\title{
Contemporary equipment in enamel process innovation design accessories
}

\author{
Luo $\mathrm{Mi}^{1, \mathrm{a}}$ \\ ${ }^{1}$ Jiangxi Institute of Fashion Technology, Jiangxi, Nanchang, 330201 \\ a124522949@qq.com
}

Keywords: Enamel craft; Accessories; Decoration; Bags; application

\begin{abstract}
In recent years, with the rapid development of creative industry, fashion bags has become one of the important element in the accessories. Especially for women dress collocation, the choice of luggage is indispensable link. Luggage industry market development prospect of huge potential, bags designer from the pursuit of innovation in enclosure structure, modelling to detail design, gradually introduce Chinese traditional culture elements, design has a unique charm and the cultural connotation of bags. This paper mainly discusses the Chinese traditional technology of enamel craft is introduced into the luggage accessories design, and analyses the enamel parts in bags modelling, colour, craft this oh domestic innovative applications, prospects the enamel accessories decorative elements in the luggage industry application prospect.
\end{abstract}

\section{The introduction}

Contemporary accessories bags in the design of various materials, although small, but to enhance the beauty of the inclusions plays an important role. Nowadays the design of equipment accessories is developing toward the direction of diversification, luggage accessories design is given priority to with metal material, plating, chromium, copper, stainless steel and other metal fittings occupy the dominant position. Because of the metallic parts will always give a person with gorgeous, noble feeling. But as people living standard rise, people from the pursuit of noble magnificent psychological turning to pursue personality avant-garde replaced, always want to own back bag is different, the modern personality. The treasure of Chinese traditional quintessence, enamel, because of its unique charm and cultural value, some handbag designer to apply enamel craft accessories in the bag, the design of the products refreshing, market potential is tremendous. At present, enamel craft accessories has been gradually used in bag design, overseas has appeared very delicate enamel equipment accessories, such as ROBERTO "black and white enamel female bag, BIBIGI enamel diamond ornamental design of female bag, etc. Enamel craft accessories for handbag designer provides a new space of art imagination.

\section{Enamel craft application feasibility in the bag}

The uniqueness of material performance.Enamel refers to the preparation of the metal material outside of the glass material, generally is the application of metal material is gold, silver, precious metals such as copper, in the ancient world belongs to the noble use items. Enamel craft such as painting, silk and pervious to light inlay enamel, process design in China Industry after the application is a kind of innovation of technology, known as "soft enamel craft, is" cloisonne craft and the product of the combination of "enamel" process.

Modern enamel glaze is a resin polymer used in the production, corrosion resistance, abrasion resistance, good color preservation integrated features, such as and glaze color category is rich, and excellent adhesion, toughness, large area coverage also won't appear in the metal surface cracking phenomenon. Modern enamel glaze has good transparency, allowing designers to adopt "painting enamel" process performance colour and texture of various kinds of graphics. At the same time, modern enamel glaze can be attached on the surface of light materials, reduce the weight of the enamel accessories, also reduces the production cost of accessories. Modern enamel of superior performance for luggage accessories laid the foundation of superior, enrich the handbag designer creative thinking. 
Affordable technology. Modern enamel have greater improvement in technology, and production can be measured. Computer drawing software may be used for the design of the product design, greatly improving the production efficiency, and to design the modified at any time, to improve the traditional wire inlay enamel in pattern design and drawing speed. And modern glaze resin's no need for a long time in high temperature furnace sintering color, as long as the chemical liquid color daub on the metal surface can present a beautiful color, baking in the oven again to finalize the design, production process is simplified. Modern enamel process of quantitative technology has greatly increased the production efficiency of equipment accessories businesses, promote the development of the equipment accessories industry, also give colour, design and modelling of modern enamel accessories bring richness, luggage accessories production for the Chinese elements provide technical assurance.

The personalized aesthetic style. Modern bags enterprises already have a strong sense of brand, some bags enterprises gradually from the pursuit of product modeling and design to the direction of art aesthetic, personality innovation and development. Modern enamel craft accessories itself is a kind of innovation, an art, because enamel technology is a comprehensive art, integrating a variety of process solutions, technologies and materials, as well as traditional Chinese national culture connotation enough. Modern enamel craft accessories into the traditional national culture, the traditional Chinese auspicious patterns such as flowers, dragons and phoenixes are used in the design, presents the elegant modelling, colour and style. Enamel craft inherit and carry forward the Chinese traditional culture and art, the modern bags designer should be to capture the information, the bag product design with Chinese characteristics. Nowadays, some high-end luggage brands abroad have enamel parts used in the design, and succeeded, is worthy of reference for Chinese handbag designer and learning. The enamel of Bvlgari, bulgari brand such as Italy head shape handbags and snake model handbags, combination of colors, materials and details using the acme, perfectly shows the Bvlgari brand bags design style, as shown in figure 1, 2.

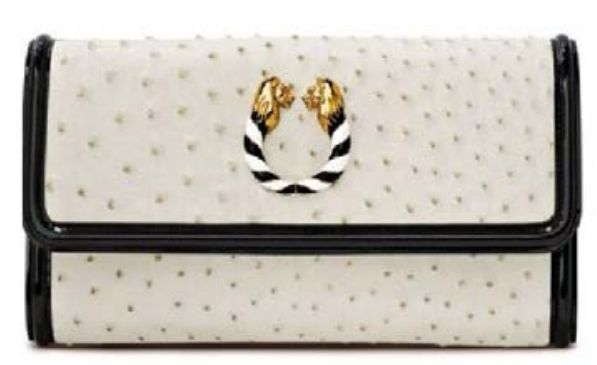

Figure 1 Enamel head shape handbags

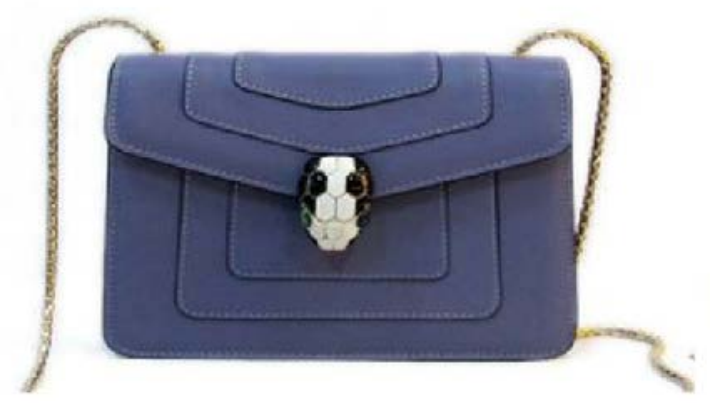

Figure 2 Enamel snake bag shape

\section{Equipment enamel craft innovation design accessories}

The application of the enamel parts of Chinese style in bag design, should notice to enamel accessories on the traditional process of innovation, the innovation, the innovation research of modelling, and its accessories design concept is to the Chinese traditional style into the luggage accessories development, perfect show the package style, character and personality elements, in order to make a handbag brand of famous brand effect.

The innovation of the enamel craft.The design and manufacture of modern enamel craft accessories mainly mechanical processing production, in the production process and the traditional wire inlay enamel products has obvious difference. Enamel craft is introduced into the modern computer intelligent design pattern, than the traditional artificial depict design saves the manpower, but also has provided the safeguard for the richness of the enamel designs, traditional point in the process of enamel glaze color is mainly artificial completed, after the high temperature of enamel glaze in the material qualitative, colorific variability is larger, and go through complementary fire to finish again, process trival. In modern enamel glaze in a traditional glaze developed on the basis of the synthetic resin glaze, safeguard the accessories of color richness and vividness. Combined with the traditional high temperature baking process of enamel process, modern enamel craft adopt 
intelligent control oven high temperature curing finalize the design, improve the production of enamel parts process speed. Traditional enamel craft is artificial buffing, polishing machine production taken now, enamel accessories elegant sex get promoted.

The innovation of the modelling of the enamel. Equipment accessories market in common shape is round, oval, triangle, square and so on, its modelling is normalized, generalization, lack of unique sense of innovation, modelling and luggage accessories combinations were arranged by way of symmetry and balanced, easy to produce aesthetic fatigue. To the need of enamel modeling innovative design, we can take in the enamel parts design point, line and plane constitute a form of creative design, design elegant, chic, glamorous handbag products. As shown in figure 3 is to use the rule of design of planar bags enamel parts, the overall effect of the female bag is beautiful. Enamel accessories can also be in the design of traditional Chinese flowers design modelling application among them, because of the enamel colour can achieve richness, so with colorful enamel inclusions color can produce a strong visual contrast, design novel, individual character of suitcases and bags, as shown in figure 4.

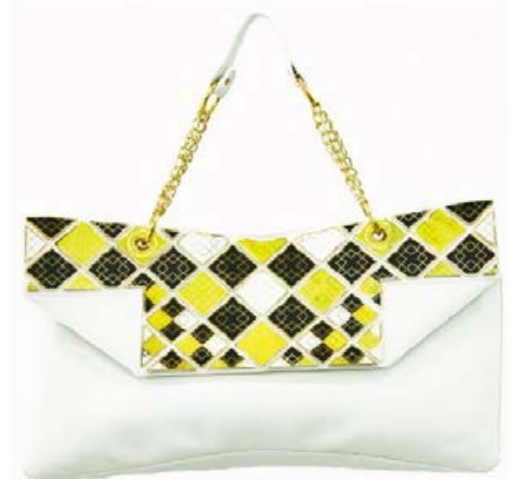

Figure 3 Shining enamel bag cover handbags

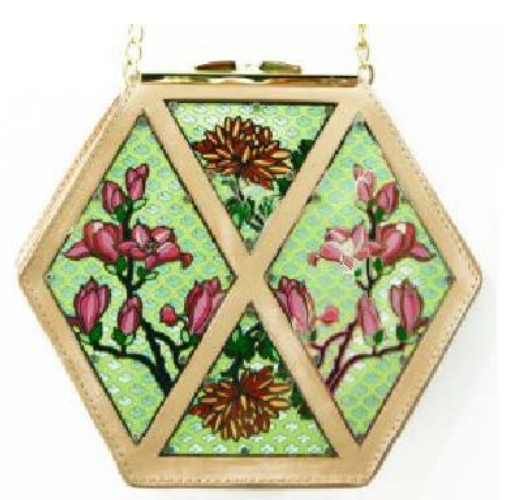

Figure 4 Peach blossom pattern charm bag bag

The exploration of the enamel color. The collocation of color is more important in the design of equipment accessories. Traditional enamel colour is single, mainly in the gold-plated, silver and other metal surface color, the colors of fashion. Due to the improvement of modern enamel craft and the enhancement, in the modern enamel craft can bold and ingenious use of gorgeous colors. In the design of the enamel parts, for example, can be a traditional Chinese lotus, peach blossom pattern and other auspicious patterns and use of Chinese landscape painting, combining traditional art and modern bags, endowed with modern fashion beautiful beautiful colour, bags as a whole reflects the retro and personalized features.

\section{Conclusion}

In the field of contemporary equipment accessories design, creative design, colour collocation rich parts more and more beautiful, material texture, the development of the luggage industry plays an important role, but equipment accessories designers also need to follow the pace of The Times, develop their own design innovation thinking, moral to create more unique and strong visual impact feeling. Handbag designer should also pay attention to the Chinese traditional culture elements into the design, and it is also a long history of China transmission and development of the cultural and technological skills. The enamel parts process combined with the concept of modern package design, efforts to promote brand bags the creation and development of China.

\section{Reference}

[1] Lili Guo. Soft enamel in jewelry design of innovative applications [J]. Journal of beauty with the time (in). 2015 (3):36-39.

[2] Zhonghao Li. Wire inlay enamel process "line" application research [J]. Journal of design. 2015 (11):77-79.

[3] Jianyi Hao. Enamel design method to combine with other materials [D]. Journal of Beijing 
institute of fashion technology. 2015.

[4]Li peng. The characteristics of the metal surface process with the process performance of the modern jewelry design [D]. Journal of China university of geosciences (Beijing). 2009.

[5] zhang jing. Cloisonne fashionable jewelry design research [D]. Donghua university. 2013.

Fund project: In 2014, jiangxi college humanities and social science planning youth project "based on" gan nuo culture tourism in jiangxi clothing product design research achievements, the project number: JC1423. 